\title{
Wirecard - ein Menetekel für die Wirtschaftsprüfung
}

Der Fall Wirecard ist einzigartig in Deutschland. Am 24. Juni 2020 hat das DAX-Unternehmen Insolvenz angemeldet. Kurz vor Aufnahme in den DAX am 24. September 2018, notierte die Wirecard-Aktie zu 197 Euro. Diesen Kurs hat Wirecard nie wieder erreicht. Danach ging es, stets begleitet von reger Leerverkaufstätigkeit, im Zick-Zack-Kurs bereits lange vor der Insolvenz stetig bergab. Noch nie ging ein Unternehmen bankrott, noch während es dem DAX angehörte. Dementsprechend groß ist das Unverständnis darüber, wie die aufgeblähte Bilanz solange unentdeckt bleiben konnte. Das Prüfmandat von Wirecard liegt seit vielen Jahren bei Ernst \& Young (EY). Jahr für Jahr hat EY die Wirecard-Bilanz als korrekt testiert. Erst im Juni 2020, nach einem für Wirecard negativ ausgefallenen Sonderprüfbericht des Konkurrenten KPMG, hat EY erstmals das Testat verweigert. Testierte Bilanzen sind ein Gütesiegel. Darauf verlassen sich Banken, Aktionäre und auch die Aufsicht. Dementsprechend fürstlich werden die Prüfmandate vergütet. Prüfer*innen sind hochbezahlte Expert*innen, auch weil ihr Testat Fundament für eine Vielzahl von Entscheidungen im Unternehmens- und Finanzsektor ist. Bilanzfälschung ist in Deutschland sicherlich nicht einfach. Das zeigt allein schon die überwältigend große Zahl der solide geführten und korrekt testierten Unternehmen. Aber die Täuschung gelingt immer wieder, dann nämlich, wenn trickreiche Vorstände mit krimineller Energie auf nachlässige und selbstgewisse Wirtschaftsprüfer treffen. Das war in der Vergangenheit so und das wird wohl auch in Zukunft so sein, wenn die Wachsamkeit, die der Wirecard-Skandal nun erzeugt hat, naturgemäß wieder nachlässt.

2001 musste das US-Energiehandelsunternehmen Enron Insolvenz anmelden. Enron, damals eines der größten US-Unternehmen, entpuppte sich als Multi-Milliarden-DollarBetrugssystem. Dank Mark-to-Market-Rechnungslegung konnte Enron jedes Jahr fantastisch hohe Bewertungsgewinne verbuchen, während die immer größer werdenden Verluste kunstreich in einem verschachtelten Geflecht aus außerbilanziellen Zweckgesellschaften versteckt wurden. Ähnlich wie bei Wirecard dienten die vielen Ableger vor allem dem einen Zweck, die wahren Zahlungsflüsse zu verschleiern. Im Dezember 2001 fiel das Kartenhaus Enron zusammen. Zu Hochzeiten war die Aktie mit über 90 US-\$ gehandelt worden. Am Tag der Insolvenz war sie keine $30 \mathrm{Cent}$ mehr wert. Geprüft und testiert wurde die Enron-Bilanz von Arthur Andersen, damals eine der größten Partnerschaften weltweit. Wie Wirecard kam auch Enron immer wieder an frisches Geld, weil sich Banken und der Kapitalmarkt auf die Testate der Wirtschaftsprüfer verließen. Sie finanzierten Enron weiter, obwohl der Geschäftsbetrieb in Wirklichkeit längst defizitär und die Rückzahlung illusorisch war. Arthur Anderson war zwar nicht selbst am Bilanzbetrug beteiligt, prüfte aber fahrlässig nachlässig. Zudem vernichtete Anderson noch interne Dokumente zu den Enron-Prüfungen, nachdem die Ermittlungen der USBörsenaufsicht SEC bereits eingesetzt hatten. Dafür wurde Anderson 2002 wegen Behinderung der Justiz verurteilt. Der Skandal um Enron brachte den Prüfgiganten zu Fall. Kurz nach dem Urteil spaltete sich Arthur Andersen in einzelne Landesgesellschaften auf. Der deutsche Ableger fusionierte zum größten Teil mit Ernst \& Young. Übrig blieb ein noch engeres Oligopol. Der lukrative Markt für Prüfmandate liegt seitdem fast vollständig in den Händen der sogenannten Big four: Deloitte, EY (Ernst \& Young), KPMG und PricewaterhouseCoopers (PwC). Der Skandal um Enron war auch Auslöser für ein neues Gesetz, dem sogenannten Sarbanes Oxley Act. Damit wurde die US-Gesetzge-

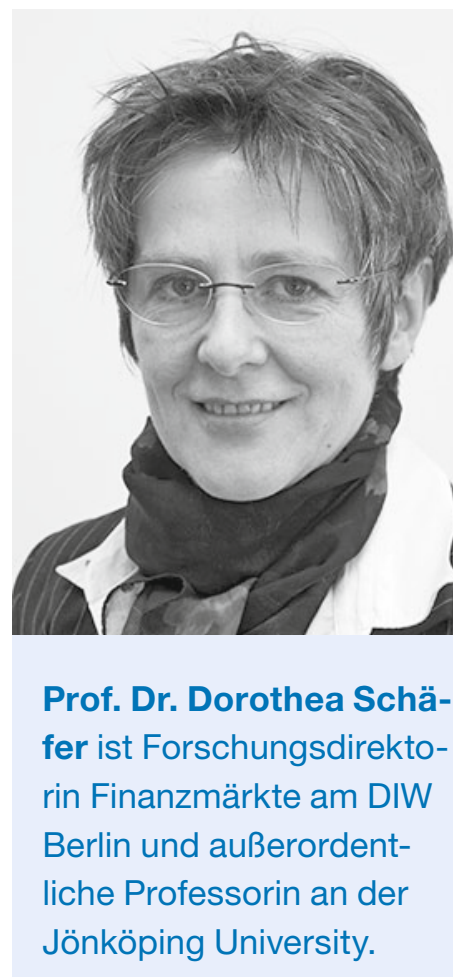

Prof. Dr. Dorothea Schäfer ist Forschungsdirektorin Finanzmärkte am DIW Berlin und außerordentliche Professorin an de 
bung zur Rechnungslegung und zum Anlegerschutz deutlich verschärft. Wirtschaftsprüfungsgesellschaften ist es seitdem beispielsweise verboten, für Unternehmen, die sie prüfen, andere, nicht prüfungsbezogene Dienstleistungen zu erbringen. Alle fünf Jahre müssen die Prüfer wechseln. Allerdings schreibt das Gesetz nur eine interne Rotation, nicht aber den Wechsel der Prüfgesellschaft vor. Zu den zentralen Reformen gehörten auch die Verschärfung der Aufsicht über die Prüfgesellschaften und die Haftung des Managements für die Richtigkeit der testierten Bilanzen.

Wirecard und Enron haben vieles gemeinsam. Dennoch greift es zu kurz, nun die US-Rechnungslegungs- und Anlegerschutzgesetze als Vorbild zu preisen. Weder der Sorban Oxley Act noch die US-Börsenaufsicht SEC konnten die Milliardenschäden durch die betrügerische Vermögensverwaltung von Berni Madoff verhindern. Auch die vielen Bilanzunregelmäßigkeiten im US-Bankensektor auf dem Weg zur Großen Finanzkrise 2008/2009 blieben unentdeckt. Das US-Finanzsystem ist zu verschieden von den Finanzsystemen kontinentaleuropäischer Prägung, als dass die dortigen Regelungen Blaupausen für hiesige Reformen sein könnten. Deutschland und die europäische Union müssen ihren eigenen Weg aus der Wirecard-Misere finden. Es wäre viel gewonnen, wenn Prüfgesellschaften, deren Kontrollorgane, Bundesanstalt für Finanzdienstleistungsaufsicht (BaFin), Deutsche Bundesbank, kreditgebende Banken und Börsen sowie Anleger*innen sogenannten „Whistleblowern“ mehr Aufmerksamkeit schenken würden. Diese Personen tragen ein hohes Risiko und verdienen schon allein deswegen ernst genommen zu werden. Alle, auch Medien und Politik, sollten jungen Fintechs mit gesundem Misstrauen begegnen, anstatt reflexartig, und mit einigem Aufwand, nach entlastenden Erklärungen für die eigenen Irritationen zu suchen. „Wachstumsstories“, die fast zu schön sind, um wahr zu sein, sind meist auch nicht wahr. Über Jahre anhaltende, äußerst rege Leerverkaufsaktivität kann ein Warnzeichen sein. Leerverkäufe lohnen sich, wenn die geliehenen Aktien teuer verkauft, und nach Kursabsturz möglichst günstig wieder erworben und an den Leihgeber zurückgegeben werden können. Je stärker der Kurs schwankt, desto attraktiver ist eine Aktie für Leerverkaufende und Leihgebende. Die Leerverkäufer sehen erhöhte Gewinnchancen. Die verleihenden Aktionär*innen freuen sich über hohe Leihgebühren. Diese Konstellation ist allerdings auch brisant. Altaktionäre mit Zugang zu Insiderwissen haben keinen Anreiz, eigenes Wissen über die wahre Lage des Unternehmens mit Aufsicht und Kleinanlegern zu teilen. Im Gegenteil, via Leihgebühren profitieren sie davon, wenn der Handel weitergeht, und der letztlich unvermeidbare Kurssturz weit in die Zukunft verschoben wird. Aufsicht ebenso wie Anleger*innen müssen die Stärke dieses Anreizes richtig einschätzen können. Daher sollten Leihgebühren, Leihgebende und Leihvolumen zusammen mit den bereits jetzt zugänglichen Leerverkaufspositionen veröffentlich werden.

Eine sehr viel höhere Haftung der Wirtschaftsprüfer würde deren Wachsamkeit zwar schärfen, aber auch die ohnehin schon extreme Konzentration weiter erhöhen. Dieses Trade-offs muss sich der Gesetzgeber bewusst sein. Stärkere Haftung sollte daher immer auch mit Maßnahmen kombiniert sein, um das enge Oligopol der Prüfgesellschaften aufzubrechen. Hätte eine durchgriffsstarke, gemeinsame Börsenaufsicht für den Euroraum den Wirecard-Betrug verhindern können? Sehr wahrscheinlich ist das nicht. Auch eine „Euro-SEC“ hätte sich wohl auf die Testate von EY verlassen. Gegenüber den asiatischen Ländern, in denen sich offensichtlich der Großteil der Unregelmäßigkeiten zugetragen hat, hätte auch die Euro-Börsenaufsicht kein Durchgriffsrecht gehabt. Dennoch ist eine gemeinsame Börsenaufsicht für den Euroraum ein Schritt in die richtige Richtung. Der Ruf nach politischer Unabhängigkeit einer

Dorothea Schäfer

DIW Berlin und Jönköping University dschaefer@diw.de zukünftigen „Euro-SEC“ ist allerdings verfehlt. Aufsichtspolitik ist anders als Geldpolitik. Auch eine gemeinsame Euro- oder EU-Wertpapieraufsicht muss der parlamentarischen Kontrolle unterliegen. 